Raf Vanderstraeten

\title{
Disziplinbildung. Zum Wandel wissenschaftlicher Kommunikation in der Soziologie
}

\section{Einleitung 1}

Wissenschaftliche Spezialisierung scheint zuerst eine intellektuelle Ausrichtung einzelner Individuen zu sein. Sie beruht auf der Entscheidung, sich auf ein relativ kleines Feld wissenschaftlicher Forschung zu konzentrieren. Wie es aber für jede Entscheidung dieser Art der Fall ist, brauchen Individuen einen sozialen Kontext, der sie unterstützt; sie brauchen andere Individuen, welche die gleiche Entscheidung treffen. Um die Mitte des 18. Jahrhunderts, als enzyklopädische Orientierungen sowohl unter professionellen Wissenschaftlern als auch unter gentlemen scientists dominierten, waren Entscheidungen für bestimmte Spezialisierungen noch selten. Sie gewannen allerdings in den letzten Jahrzehnten des 18. Jahrhunderts und zu Beginn des 19. Jahrhunderts an Bedeutung.

Es ist bekannt, dass die Ausdifferenzierung von wissenschaftlichen Spezialisierungen mit der Formierung von scientific communities, von Netzwerken von Spezialisten einhergeht (siehe z. B. Kuhn 1962). Aber wie findet die Formierung solcher Netzwerke statt? Und wie wird eine kollektive Orientierung der Mitglieder einer scientific community aufrechterhalten? In einer Reihe von Schriften hat Rudolf Stichweh die Aufmerksamkeit auf die Entstehung spezifischer Formen wissenschaftlicher Kommunikation gelenkt und insbesondere die Rolle wissenschaftlicher Zeitschriften betont. Wie Stichweh hervorhebt, machen wissenschaftliche Fachzeitschriften neue Formen von Kommunikation möglich und fördern die Entstehung von Netzwerken potenzieller $\mathrm{Au}$ toren und Spezialisten. Zeitschriften und die darin erscheinenden Veröffentlichungen strukturieren die wissenschaftliche Kommunikation. Die Autoren von Aufsätzen akzeptieren die von der Zeitschrift gewählte Spezialisierung, modifizieren aber gleichzeitig diese Spezialisierung durch den kumulativen Effekt ihrer veröffentlichten Ergebnisse. Die wissenschaftlichen Zeitschriften bilden ein Medium, durch welches Kommunikationskomplexe, die entlang disziplinärer Linien spezialisiert sind, verbunden und auf Dauer bestehen

1 Ich danke Brigitte Lohan für ihre Hilfe bei der Endredaktion dieses Aufsatzes. 
werden. Sie ermöglichen die Selbstorganisation und Autopoiesis wissenschaftlicher Disziplinen (Stichweh 1984, 394 ff.; 1987; 1994, 14 ff.; siehe auch Luhmann 1990, 271ff.).

Mehr noch als Bücher zeigen spezialisierte Zeitschriften die kollektiven Strukturen der wissenschaftlichen Kommunikation. Es ist wahrscheinlich, dass die Zeitschrift die theoretische und methodologische Variabilität einer (Sub-)Disziplin abbildet, da sie aus einer Sammlung verschiedener Aufsätze von verschiedenen Autoren besteht. Dabei kann gerade die Diversität der veröffentlichten Beiträge eine Reflektion über die Beziehung zwischen den Beiträgen und ihren Zusammenhang hervorrufen. Mehr als von Büchern wird von einer Zeitschrift erwartet, dass sie ein aktuelles Bild eines gesamten Forschungsfeldes präsentiert. Von jedem Zeitschriftaufsatz wird auch erwartet, dass er auf die vorhergehenden Bezug nimmt, indem er Argumente mit einbezieht, die in anderen Aufsätzen bereits entwickelt wurden. Aufgrund der Ansprüche, die er auf neues Wissen erhebt, fordert jeder neue Aufsatz auch Reaktionen und somit weitere neue Aufsätze heraus. In diesem Sinne stehen Publikationen in spezialisierten Zeitschriften für die Kontinuität disziplinärer Kommunikationsprozesse. Solche Publikationen - erst eingebunden in nationale, danach zunehmend in supranationale Netzwerke - sind von zentraler Bedeutung für die Formierung von intellektuellen Spezialisierungen und wissenschaftlichen Disziplinen. Sie bilden heutzutage die privilegierte, wenn nicht gar die kanonische Form wissenschaftlicher Kommunikation. Analysen der Evolution dieser wissenschaftlichen Publikationen können daher auch zu unserem Verständnis der Identität und der Evolution der Identität wissenschaftlicher Disziplinen und Spezialisierungen beitragen. ${ }^{2}$

Wenn man wissenschaftliche Disziplinen als Kommunikationsnetzwerke begreift, dann stellt sich auch die Frage, wie disziplinäre Kommunikationsprozesse erzeugt und organisiert werden. Ich möchte dieser Frage empirisch nachgehen, und dabei zwei Themen behandeln, mit denen sich auch Rudolf Stichweh immer wieder befasst hat (siehe Stichweh 2003a; 2003b). Auf der Basis einer Fallstudie über die wissenschaftliche Kommunikation in soziologischen Zeitschriften in den Niederlanden und dem nördlichen Teil von Belgien werde ich mich einerseits mit der Evolution von Inklusionsformen, andererseits mit (fehlender) Globalität befassen. Dazu folgt zunächst eine kurze Skizze der Landschaft soziologischer Zeitschriften in den Niederlanden und Belgien. Danach werden empirische Daten, die längerfristige Veränderungen in den Kommunikationspraktiken innerhalb der niederländischsprachigen Soziologie beschreiben, präsentiert und diskutiert.

2 Die bibliometrischen und scientometrischen Instrumente die in den letzten Jahrzehnten entwickelt wurden, wie zum Beispiel ISI's Journal Citation Reports und Journal Performance Indicators, haben die Bedeutung von Zeitschriften im wissenschaftlichen Kommunikationsprozess sicherlich verstärkt. 


\section{Soziologische Zeitschriften in den Niederlanden und Belgien}

Die ersten Zeitschriften, die sich auf Soziologie spezialisierten, tauchten in den letzten Jahrzehnten des 19. Jahrhunderts auf. Unter ihnen war die Revue Internationale de Sociologie, gegründet 1892 von René Worms (inzwischen besser bekannt unter ihrem englischen Titel International Review of Sociology), das American Journal of Sociology, gegründet 1895 als die erste US-amerikanische wissenschaftliche Zeitschrift in der Disziplin und die L'année Sociologique, gegründet u.a. von Émile Durkheim, welche erstmals 1898 erschien. Seit dieser Zeit wurden viele weitere soziologische Zeitschriften in fast allen Teilen der Welt gegründet. ${ }^{3}$ Nicht alle diese Zeitschriften existieren noch. Einige von ihnen wurden bereits nach wenigen Heften nicht mehr herausgegeben; andere haben länger existiert, aber verschwanden dennoch - zum Beispiel als Folge eines Manuskriptmangels oder finanzieller Probleme. Aber insgesamt liefern uns diese Zeitschriften und ihre Geschichte reichhaltiges Material für die soziologische Analyse der Morphogenese der Soziologie selbst.

Im 20. Jahrhundert entstanden auch im niederländischen Sprachraum eine Anzahl soziologischer Zeitschriften. 1925 erschien die erste (proto-)soziologische Zeitschrift in den Niederlanden: Mensch \& Maatschappij (d. h. »Mensch \& Gesellschaft«, im Folgenden M\&M). Ursprünglich hatte M\&M einen thematisch sehr breiten Fokus. Der erste Untertitel zählte elf (Sub-)Disziplinen auf, die sich mit dem Verhältnis von Mensch und Gesellschaft beschäftigten: Anthropologie, Ethik, Ethnologie, Eugenik und Genetik, Psychologie, Sozialgeographie, Soziologie, usw. Offiziell gab es zudem keinen geschäftsführenden Herausgeber; die Editorials wurden unterzeichnet von »den Herausgebern" (von welchen es zu Beginn ebenfalls elf gab). Trotzdem zeichneten sich bereits zu Anfang bestimmte Schwerpunkte im Inhalt von M\&M ab. So war der Hauptartikel der ersten Ausgabe einer Diskussion neuer Entwicklungen innerhalb der deutschen Soziologie gewidmet. Sein Autor war Sebald Steinmetz, welcher der Generation von Durkheim und Weber angehörte und bereits seit ca. 1890 mehrere Plädoyers für eine »soziographische« Soziologie veröffentlicht hatte (auch regelmäßig in deutscher Sprache). Im Laufe der Zeit grenzte die Zeitschrift ihr Spektrum dann ganz offensichtlich ein. Ende der 1950er Jahre wurde dies formell bestätigt, indem der ursprüngliche Untertitel der Zeitschrift verschwand. Seit dieser Zeit präsentiert sich M\&M selbst als eine allgemeine Zeitschrift für die Sozialwissenschaften stricto sensu, je-

3 In Deutschland gab es ähnliche Initiativen: 1904 übernahmen Max Weber, Edgar Jaffé und Werner Sombart die Redaktion des Archivs für Sozialwissenschaft und Sozialpolitik (die Zeitschrift war die Nachfolgerin des von Heinrich Braun begründeten Archivs für soziale Gesetzgebung und Statistik). 1933 stellte diese Zeitschrift ihr Erscheinen aber ein. Die Kölner Vierteljahrshefte für Sozialwissenschaften und die Kölner Vierteljahrshefte für Soziologie wurden zwischen 1921 und 1934 publiziert; 1948 wurde die Kölner Zeitschrift für Soziologie und Sozialpsychologie gegründet. Die Soziale Welt erschien zuerst 1949; die Zeitschrift für Soziologie wurde 1972 gegründet. Soziale Systeme entstand 1995. 
doch mit einer starken quantitativen Orientierung. Ungeachtet einer Reihe von Problemen, sowohl redaktioneller als auch finanzieller Art, ist M\&M bis heute, also mehr als acht Jahrzehnte ununterbrochen erschienen.

Der Sociologische Gids (d.h. Soziologischer Führer; im Folgenden SG) wurde 1953 von jungen Absolventen der Universität von Amsterdam gegründet. Diese Zeitschrift war nicht nur ein Produkt der Expansion der Sozialwissenschaften nach dem zweiten Weltkrieg, sie trieb die Ausdifferenzierung der Soziologie auch voran. Von Beginn an wählte ihre Herausgeberschaft einen explizit disziplinären Fokus. Es war die erste Zeitschrift in den Niederlanden, die ausdrücklich darauf abzielte, zur Professionalisierung der Soziologie beizutragen (siehe auch Wilterdink/Van Heerikhuizen 2004). Die Zeitschrift verstand sich als eine allgemeine soziologische Fachzeitschrift, die Beiträge aus allen Forschungs- und Themenbereichen der Soziologie veröffentlichte. In der zweiten Hälfte des 20. Jahrhunderts operierten SG und M\&M nebeneinander. M\&M wurde mehr und mehr zu einem Sprachrohr der methodologischquantitativen Forschung in der Soziologie, SG dagegen blieb ungefähr ein halbes Jahrhundert lang eine Zeitschrift, die vor allem Beiträge zu allgemeinen Themen und Forschungsbereichen der Soziologie veröffentlichte. 2004, kurz nach der Feier des fünfzigsten Jahrestages ihrer Erstveröffentlichung, wurde die Publikation dieser Zeitschrift dennoch eingestellt. Wie am Anfang des 21. Jahrhunderts wiederholt in den Geschäftsberichten festgestellt wurde, zwang der anhaltende Mangel an (qualitativ hochwertigen) Einreichungen die Herausgeber, die Veröffentlichung von SG in seiner damaligen Form zu beenden.

In der zweiten Hälfte des 20. Jahrhunderts wurden einige weitere, subdisziplinär spezialisierte niederländischsprachige Zeitschriften gegründet, zum Beispiel im Bereich der Sozialpolitik. Andere neue, eher allgemein orientierte soziologische Zeitschriften waren eng an bestimmte Universitäten und ihre Traditionen angebunden. Es war eine von diesen, die Amsterdams Sociologisch Tijdschrift, mit der SG sich 2004 zusammenschloss. AST war eng verbunden mit der Amsterdam School for Social Science Research und ihrer (oder Norbert Elias') Figurationssoziologie. 2004 hatte diese Zeitschrift gerade ihr 30-jähriges Bestehen gefeiert; sie sah sich aber mit ähnlichen Problemen wie SG (und fast alle anderen niederländischsprachigen Zeitschriften) konfrontiert. Die neue Zeitschrift, die aus SG und AST entstanden ist, trägt den einfachen Titel Sociologie.

In Belgien ist die scientific community (im Vergleich zu den anderen Ländern Europas) relativ klein und heterogen. Soziologische Fachzeitschriften wurden hier erst ziemlich spät gegründet. Dies ist sowohl eine Konsequenz der Divergenz zwischen dem niederländischsprachigen und dem französischsprachigen Teil (d.h. zwischen Flandern und der wallonischen Region) Belgiens, als auch eine Konsequenz der ideologischen Spannungen zwischen den verschiedenen `Säulen< (katholisch oder nicht) und ihren Universitäten (Vander- 
straeten 1999). Obwohl es in den 1960er Jahren möglich wurde, einen Universitätsabschluss in Soziologie in Flandern zu machen, wurde hier die erste und bisher auch die einzige allgemeine niederländischsprachige soziologische Zeitschrift erst 1979/1980 gegründet: die Tijdschrift voor Sociologie (d. h. Zeitschrift für Soziologie, TvS). ${ }^{4}$ Diese Zeitschrift besteht jetzt also ungefähr 30 Jahre. In dieser Zeit hat sie aber auch wiederholt mit gravierenden Problemen hinsichtlich Einreichungen und Abonnenten Erfahrung gemacht (Jacobs/De Wit 2004). Wie wir später noch im Detail sehen werden, bereitet die Fokussierung auf eine globale Orientierung der wissenschaftlichen Kommunikation für die niederländischsprachigen Zeitschriften in Belgien sowie in den Niederlanden selbst erhebliche Probleme.

Im nächsten Abschnitt wird eine detaillierte empirische Analyse der Kommunikation innerhalb der niederländischsprachigen Soziologie präsentiert. Es scheint mir unumstritten, dass Wissenschaftler darauf trainiert sind, ihre eigene Arbeit und die Arbeit anderer kritisch zu beobachten. Aber diese kritische Energie ist fast vollständig auf den wissenschaftlichen Produktionsprozess gerichtet, auf die stechnischen $<$ Aspekte von Forschung. Die Kommunikationsaspekte werden viel weniger beachtet. Die Formen wissenschaftlicher Kommunikation werden in der Regel als gegeben angesehen. Sie werden meist nicht als sozial und historisch spezifische Formen verstanden, die Druck auf den Forschungsprozess ausüben und die Bedingungen für die Inklusion im disziplinären Kommunikationsprozess festlegen. Dennoch tragen und lenken die Fachzeitschriften den Kommunikationsprozess innerhalb wissenschaftlicher Disziplinen. Und sie tun dies in einer Art, die vorstrukturiert, wie $\mathrm{Au}-$ toren Beiträge zum state-of-the-art bestimmter wissenschaftlicher Disziplinen beisteuern können. Vor diesem Hintergrund sollen im Folgenden einige allgemeine und spezifische Charakteristika des Wandels der wissenschaftlichen Kommunikation in der niederländischsprachigen Soziologie detaillierter besprochen werden. Wie bereits im ersten einführenden Absatz kurz erwähnt, werde ich mich dabei einerseits mit dem Wandel von Inklusionsformen (insbesondere in Leistungsrollen), andererseits mit der globalen Orientierung der wissenschaftlichen Kommunikation in diesen Zeitschriften näher befassen.

4 Im französischsprachigen Teil von Belgien entstand 1970 die Zeitschrift Recherches Sociologiques. Sie wurde gegründet und herausgegeben von Mitgliedern der katholischen Universität von Louvain-la-Neuve (UCL). Obwohl ihr >offener Geist betont wurde, hatte die Zeitschrift Probleme, ihre lokale ideologische Einbindung zu übersteigen. Unter dem Druck nachlassender Einreichungen und Abonnenten fusionierte man 2005 mit einer lokalen anthropologischen Zeitschrift. Für die neue Zeitschrift hat man den Titel Recherches Sociologiques et Anthropologiques gewählt. Insgesamt existierten und existieren nur wenige Kontakte zwischen den niederländischsprachigen und den französischsprachigen Soziologen in Belgien. Sprache stellt die Basis dar, auf welcher die scientific communities aufgebaut sind. 


\section{Autoren und Aufsätze}

Die soziologischen Fachzeitschriften in den Niederlanden und Belgien operierten von Beginn an in einem globalen Kommunikationskontext. In vielen anderen Ländern wurden ähnliche Fachzeitschriften mit ähnlichen Zielen gegründet. Für die Sozialwissenschaften blieb die disziplinäre Kommunikation bis zur zweiten Hälfte des 20. Jahrhunderts dennoch überwiegend auf nationalem Niveau organisiert. Im Vergleich zu anderen national communities bildet die niederländischsprachige Soziologie ein relativ kleines und peripheres Kommunikationsnetzwerk.

Ein Anzeichen für den peripheren (oder semi-peripheren) Status der niederländischsprachigen Zeitschriften in der Weltwissenschaft ist die Tatsache, dass sie nicht im Web of Science und einem seiner Indizes (wie zum Beispiel den Social Science Citation Index oder SSCI) erfasst sind. Diese Datenbanken haben eine klare Ausrichtung auf englischsprachige Zeitschriften. Zwar wurden M\&M, SG und TvS in das Verzeichnis Sociological Abstracts aufgenommen, aber die Erfassung des Inhalts ihrer älteren Jahrgänge ist hier oft unvollständig und irreführend. Ich habe daher eine neue Datenbank zusammengestellt - ausgehend von den einzelnen Heften dieser Zeitschriften selbst. Diese Datenbank enthält alle Publikationen, die in diesen Zeitschriften erschienen sind, mit Ausnahme von Forschungsnotizen, Buchbesprechungen, Konferenzberichten, Nachrufen/Ehrungen, Editorials und Einleitungen zu Themenheften. Die Datenbank umfasst insgesamt 3264 Publikationen aus dem Zeitraum zwischen 1925 und 2010. ${ }^{5}$

Abbildung 1 zeigt für jede der oben genannten Zeitschriften die Entwicklung der durchschnittlichen Anzahl von Autoren pro veröffentlichten Aufsatz - allerdings mit dreijährlichen gleitenden Durchschnittswerten. Von den insgesamt 3264 Aufsätzen sind 2477 (d.h. $75.9 \%$ ) von einem einzigen Autor verfasst worden. Veröffentlichungen mit mehreren Autoren waren bis in die 1960er Jahre die Ausnahme, sind aber in den letzten Jahrzehnten zunehmend üblicher geworden (siehe auch De Haan 1997). 1965 noch war jeder M\&MAufsatz single-authored. 1985 waren 50 \% von einem einzigen Autor, während 2005 oder $201081 \%$ der Aufsätze von mehreren Autoren verfasst waren. Die durchschnittliche Anzahl von Autoren pro M\&M-Aufsatz stieg von 1.0 (1925 oder 1965) auf 1.6 (1985) und 2.3 (2005) und 2.5 (2010) an. Für SG und TvS sind die Daten und Trends nicht sehr unterschiedlich (ungeachtet einiger relativ starker Fluktuationen). Auch hier dominieren single-authored Aufsätze die

5 Der Schwerpunkt dieser Diskussionen liegt bei Aspekten, die am besten vor dem Hintergrund eines umfangreichen Bestandes quantitativen Materials über Publikationen zu betrachten sind (siehe auch Keiner 1999). Dennoch habe ich auch verschiedene individuelle Publikationen genauer untersucht, wie etwa die Editorials aller Hefte. Außerdem hatte ich Zugang zu den Archiven einer der Zeitschriften (TvS). Ich berufe mich hier nicht auf dieses qualitative Material (vor allem aus Platzgründen). Aber einige Entscheidungen, die bei der Präsentation des quantitativen Materials getroffen wurden, sind von ihm beeinflusst. 


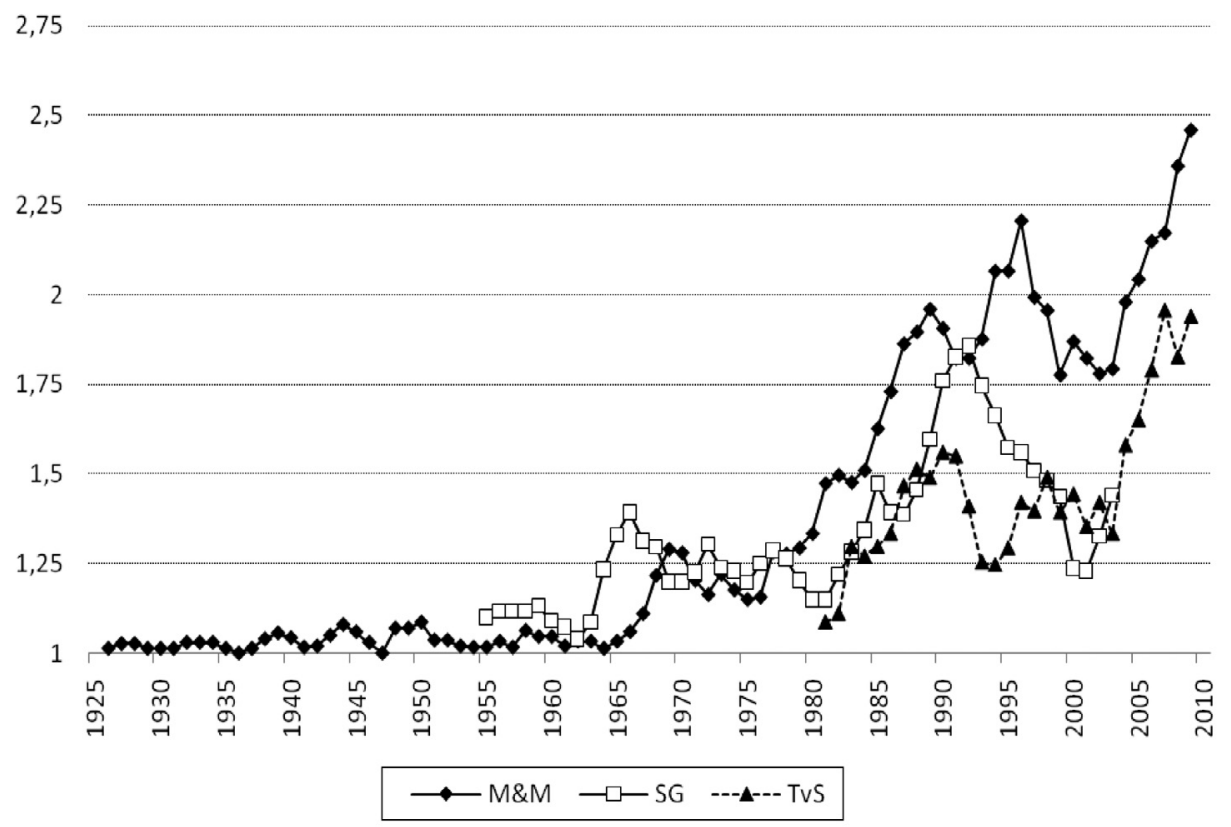

Abbildung 1: Durchschnittliche Anzahl Autoren pro Aufsatz (dreijährliche gleitende Durchschnittswerte)

ersten Ausgaben, während Veröffentlichungen mit 3, 4 oder 5 Autoren in den aktuelleren Jahrgängen zunehmend üblicher werden. Sowohl die Anzahl der Aufsätze mit mehreren Autoren als auch die durchschnittliche Anzahl von Co-Autoren pro Artikel stiegen also. Als Folge dieser Entwicklung ist nun eine steigende Anzahl von Forschern in der Lage, als Autor zu wissenschaftlichen Diskussionen im Bereich der Soziologie beizutragen. In diesem Sinne wurde der Zugang zu wissenschaftlichen Kommunikationsnetzwerken und wissenschaftlichen Veröffentlichungen in den letzten Jahrzehnten erleichtert. Mit anderen Worten: die Inklusion in wissenschaftliche >Leistungsrollen< (als Autor) wird vereinfacht.

Heutzutage besteht nicht nur ein struktureller Publikationsimperativ; auch ein struktureller Druck in die Richtung von co-authored oder multiple-authored Publikationen wird institutionalisiert. 25\% der M\&M-Aufsätze die zwischen 1925 und 2010 veröffentlicht wurden, waren von mehreren Autoren verfasst gegenüber den $23 \%$ aller SG-Aufsätze und den $30 \%$ aller TvS-Aufsätze die bis 2010 veröffentlicht wurden. In Anbetracht ihrer relativ kurzen Geschichte sind SG und TvS weniger mit co-authored Veröffentlichungen vertraut. Dennoch sind Veröffentlichungen in M\&M in den letzten Jahrzehnten häufiger von mehreren Autoren verfasst und haben im Durchschnitt mehr Autoren pro Veröffentlichung als die anderen soziologischen Zeitschriften in den Niederlanden oder Belgien. Wie bereits gesagt: M\&M ist im niederländischen Sprachraum zu der 
Zeitschrift geworden, die sich hauptsächlich der Veröffentlichung empirischer, quantitativer Forschungen widmet. Diese Art von Forschung scheint am leichtesten eine Art von Arbeitsteilung zu ermöglichen (Pontille 2003).

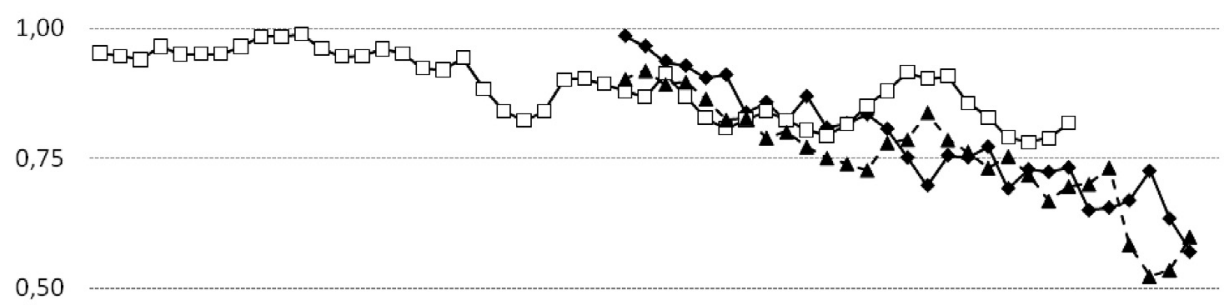

0,25

0,00

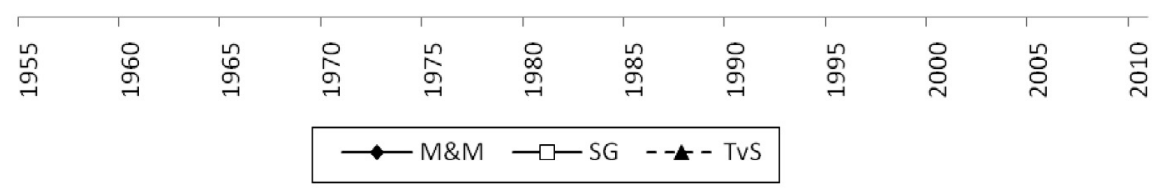

Abbildung 2: Anteil männlicher Autoren (dreijährliche gleitende Durchschnittswerte)

Um die Charakteristika der Autorschaft genauer zu beschreiben, zeigt Abbildung 2 die historischen Veränderungen hinsichtlich der Repräsentation von Frauen unter den Zeitschriftenautoren. Aufgrund der langjährigen niederländischen Angewohnheit, den Autoren nur Initialen zu geben, können hier lediglich Daten ab 1954 präsentiert werden. ${ }^{6}$ Nur 11 \% aller Autoren der SG waren Frauen. Bis Ende der 1960er hatten Frauen kaum Zugang zur Autorschaft in dieser Zeitschrift. In den letzten 20 Jahren des Bestehens von SG schwankte ihr Anteil aber zwischen 7 und $25 \%$. Der Anstieg weiblicher Beteiligung ist stärker für die Zeitschriften M\&M und TvS. In den frühen 1980er Jahren trugen beinahe keine Frauen zu diesen Zeitschriften bei. Seit Beginn des 21. Jahrhunderts jedoch sind ca. $30 \%$ der Autoren in beiden Zeitschriften Frauen. Dieser Anstieg in der Repräsentation von Frauen geht mit dem Anstieg der durchschnittlichen Anzahl von Autoren pro Artikel einher. Trotzdem sind nur wenige der Veröffentlichungen von mehreren Autoren exklusiv

6 Ich danke J.E. Ellemers, einer der Gründungsherausgeber von SG und auch ihr produktivster Autor (mit insgesamt 18 single-authored SG-Aufsätzen), der mir geholfen hat, die biographischen Informationen für eine ganze Reihe Autoren zu vervollständigen. Für SG konnten ungefähr $5 \%$ (71 von 1395) der Autoren nicht nach Geschlecht kodiert werden. Bei der Mehrzahl der Autoren der frühen M\&M-Aufsätze war es bisher unmöglich, zusätzliche Informationen über ihre Vornamen (Geschlecht) und institutionelle Zugehörigkeit zu finden. Die Abbildungen 2 und 3 umfassen daher nicht die gleiche Zeitspanne wie Abbildung 1. 
weibliche Veröffentlichungen. In TvS zum Beispiel, wirkten Frauen zwischen 1980 und 2010 an 48 \% der Aufsätze mit mehreren Autoren mit. Aber nur $9 \%$ der Veröffentlichungen von mehreren Autoren in dieser Zeit haben nicht wenigstens einen männlichen Co-Autor.

Die männliche Mehrheit unter Autoren erlaubt übrigens nicht den Schluss, dass Frauen in den Kommunikations- und Publikationsnetzwerken der niederländischsprachigen Soziologie unterrepräsentiert sind. Es gibt keine zuverlässige Auflistung von Personen die in M\&M, SG oder TvS hätten publizieren können. Es gibt Auflistungen von Lehrpersonal, angestellt an belgischen und niederländischen Universitäten, aber verlässliche biographische Daten über alle Forscher im Feld der Soziologie (von denen viele auf Basis von Kurzzeitund Teilzeitverträgen angestellt waren/sind) existieren nicht. Tatsächlich legen etwa Daten zur Soziologie in Großbritannien nahe, dass Frauen in dem dort etablierten Zeitschriftenpublikationsprozess nicht diskriminiert wurden / werden (Platt 2007).

Natürlich sind die Veränderungen hinsichtlich Autorschaft nicht nur charakteristisch für die Kommunikationspraxis in M\&M, SG und TvS. Ähnliche Trends wurden in anderen akademischen Forschungsfeldern und anderen wissenschaftlichen Netzwerken beobachtet (siehe Clark 1999; Platt 2007). In Disziplinen wie Physik, Biologie, Mathematik oder Astronomie sind wissenschaftliche Aufsätze, die nur von einem männlichen/weiblichen Autor geschrieben werden, höchst außergewöhnlich geworden. Die skizzierten Trends weisen aber auch eine spezifische Paradoxie auf.

Einerseits ist deutlich, dass wissenschaftliche Zeitschriften die Kommunikation von Forschungsergebnissen nicht nur ermöglichen. Sie beeinflussen auch, in welcher Form Beiträge zu wissenschaftlicher Kommunikation geleistet werden können. Im Vergleich mit der Produktion und dem Umlauf von Büchern führen Zeitschriften oder Periodika zu einer schnellen Abfolge kleiner Beiträge. Veröffentlichungen in Periodika folgen einander in kurzen und regelmäßigen Abständen. Zeitschriften beeinflussen die zeitliche Struktur des Wissenschaftssystems. Auf die veröffentlichten Ergebnisse eines Zeitschriftenaufsatzes kann schon im nächsten Heft der Zeitschrift reagiert werden. Die Periodizität des Erscheinens setzt Wissenschaftler unter Druck, regelmäßig zu veröffentlichen (publish or perish). Andererseits ist aber auch deutlich, dass die zunehmende Zahl von co-authored Aufsätzen und von Autoren pro Aufsatz eben die Zurechnung von Beiträgen auf Autoren nicht leichter macht. Vor dem Hintergrund des skizzierten Wandels kann man behaupten, dass es immer schwieriger wird, den Autor einer bestimmten Veröffentlichung zu identifizieren. Mit Michel Foucault (1995, 789 ff.) kann man eben von einem Verschwinden oder einer Auslöschung des Autors sprechen. Vielleicht ist tatsächlich eines der Paradoxien der spostmodernen< Gesellschaft, dass Veröffentlichungen in wissenschaftlichen Zeitschriften fast gleichzeitig viel bedeutsamer geworden sind für die Beurteilung oder Bewertung individueller Forscher und Forschergruppen. 
IV. Supranationale Kommunikationsnetzwerke

Um Aufschluss über einige der historischen Besonderheiten wissenschaftlicher Kommunikation in einem (semi-)peripheren Teil der Weltwissenschaft zu geben, bietet Abbildung 3 einen Überblick über die institutionelle Zugehörigkeit der Autoren von M\&M, SG und TvS. Diese Abbildung macht sofort deutlich, dass die große Mehrheit der Autoren - tatsächlich: fast alle - ihren Wohnsitz in den Niederlanden oder in Belgien haben. Die Präsenz von sAusländern in den 1960er und 1970er Jahren war das Resultat einiger Sonderinitiativen der Herausgeber von SG, wie zum Beispiel die Veröffentlichung von Themenheften oder Sonderbänden, bei denen diese >Ausländer zu Beiträgen eingeladen wurden. Manchmal wurden Übersetzungen von Aufsätzen von deutsch- oder englischsprachigen Autoren veröffentlicht; gelegentlich nahmen diese Zeitschriften auch englischsprachige Aufsätze auf. In den letzten Jahren haben multinationale Forschungsprojekte hier und da zu multinationalen Veröffentlichungen mit mehreren Autoren geführt, aber die führenden soziologischen Fachzeitschriften in Belgien und den Niederlanden überschritten / überschreiten in der Regel die Grenzen ihres Sprachraums nicht: M\&M, SG und TvS waren bisher kaum in der Lage, internationale Autorenschaften anzuwerben. Die langfristige Evolution von Veröffentlichungen in SG erlaubt es eben, so gesehen, von einer abnehmenden Internationalisierung der niederländischsprachigen Soziologie zu sprechen.

1

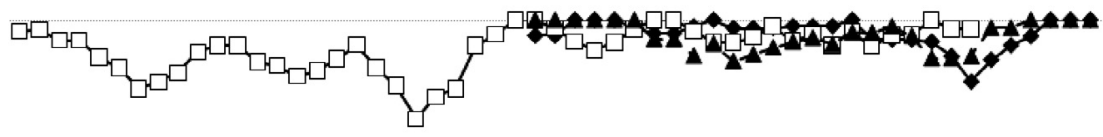

0,75

0,5

0,25

0

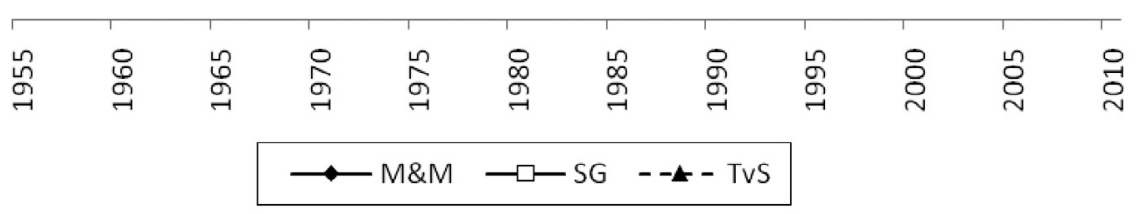

Abbildung 3: Anteil Autoren aus den Niederlanden oder Belgien 
Zusätzlich kann betont werden, dass jede dieser Zeitschriften ein relativ starkes nationales (und nicht nur sprachliches) Profil beibehalten hat. Vor der Gründung der TvS trugen nur wenige flämische Soziologen zu niederländischen soziologischen Fachzeitschriften bei. Weniger als 2\% der Autoren (12 von 648), die in SG vor 1980 veröffentlichten, kamen aus Belgien. In den letzten Jahrzehnten hat sich die Situation kaum verändert. Etwa $5 \%$ der Autoren, die zwischen 1980 und 2000 in M\&M oder SG veröffentlichten, arbeiteten zu dieser Zeit in Belgien. Eine etwas mildere Form der Exklusion besteht in die andere Richtung: $16 \%$ der Autoren (84 von 521), die ihre Arbeit zwischen 1980 und 2005 in TvS veröffentlichten, arbeiteten in den Niederlanden. Diese Beteiligung findet aber, wie bereits gesagt, nicht auf regelmäßiger Basis statt. Die Beteiligung ist vor allem unregelmäßigen Initiativen, wie der Veröffentlichung von Sonderheften mit eingeladenen Autoren zu verdanken. Ansonsten blieb jede dieser Zeitschriften relativ fest in ihren eigenen nationalen Kontext eingebettet.

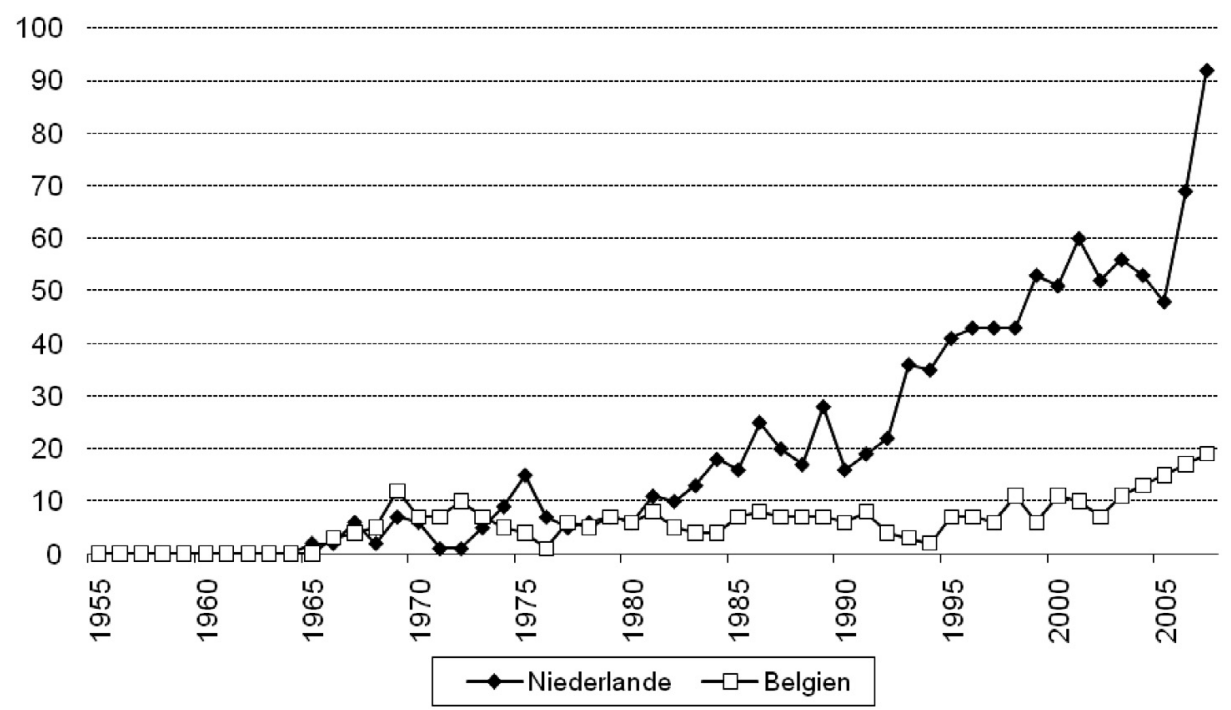

Abbildung 4: Veröffentlichungen in SSCI-Zeitschriften (absolute Zahlen)

Abbildung 4 blickt in die andere Richtung. Sie präsentiert einen Überblick über die Anwesenheit belgischer oder niederländischer Autoren in den weltweit führenden Zeitschriften im Bereich der Soziologie, nämlich in den Zeitschriften, die unter der Rubrik Soziologie der Social Science Citation Index aufgelistet sind. Es besteht ein geringer Unterschied zwischen Belgien und den Niederlanden bis in die 1970er Jahre. Dies ist einfach deshalb so, weil Soziologen in beiden Ländern kaum in den global sichtbarsten Periodika ihrer Disziplin veröffentlichten. Ab der zweiten Hälfte der 1960er Jahre wuchs 
die globale Orientierung in den Niederlanden. Der Anstieg niederländischer Veröffentlichungen in internationalen Zeitschriften ist in den letzten Jahren selbst spektakulär. Für in Belgien arbeitende Soziologen bleiben Veröffentlichungen in den SSCI-Zeitschriften weiterhin die Ausnahme (obwohl der Trend sich auch hier in den letzten Jahren zu verändern scheint). ${ }^{7}$ Einer der Hauptgründe für die bemerkenswerte Divergenz zwischen beiden Ländern ist die Wissenschaftspolitik niederländischer Förderorganisationen und Universitäten. Seit den späten 1980er Jahren ist die Anzahl von Veröffentlichungen in SSCI-Zeitschriften bestimmend für die Beurteilung und das ranking von Wissenschaftlern und wissenschaftlichen Einheiten. Die Zuweisung von Forschungsmitteln wie auch individuelle Beförderungen wurden abhängig gemacht von dieser Art Output. Aber diese Wissenschaftspolitik hatte auch eine unbeabsichtigte und unerwartete Kehrseite: sie hat den Rückgang der niederländischsprachigen Zeitschriften wenngleich nicht verursacht, so doch verstärkt. Für wen ist es noch wichtig, seine/ihre Arbeit in niederländischsprachigen Zeitschriften zu veröffentlichen, wenn diese Zeitschriften in offiziellen Bewertungen von Forschungsergebnissen nicht mitzählen? Wie viele Einreichungs- und Abonnentenprobleme der niederländischsprachigen Zeitschriften (bis hin zur Einstellung der SG)zeigen, haben diese nationalen Fachzeitschriften Probleme, sich der wachsenden Rolle supranationaler Netzwerke anzupassen.

Es ist zugleich interessant, einen genaueren Blick auf die SSCI-Zeitschriften zu werfen, in denen Arbeiten von Autoren aus Belgien und den Niederlanden veröffentlicht wurden. Niederländische Autoren ziehen klar englischsprachige Zeitschriften mit einer empirischen und/oder methodologischen Orientierung vor. Bis jetzt wurden die meisten ihrer Arbeiten in den folgenden soziologischen Fachzeitschriften veröffentlicht (in absteigender Reihenfolge): Social Indicators Research, Sociologica Ruralis, European Sociological Review, Social Networks, Journal of Marriage and Family, Sociological Methods \& Research, Journal of Mathematical Sociology, und Social Compass. Darüber hinaus existierte zwischen 1962 und 2004 eine SSCI-Zeitschrift, die englische Übersetzungen einer Auswahl von Aufsätzen veröffentlichte, die vorher in niederländischsprachigen sozialwissenschaftlichen Zeitschriften veröffentlicht worden waren. Sie sollte das >Beste< der niederländischen Soziologie für ein breiteres Publikum zugänglich machen. Die Zeitschrift erschien nacheinander unter verschiedenen Titeln: Sociologia Neerlandica, The Netherlands' Journal of Socio-

7 Eine Anmerkung muss (erneut) gemacht werden. Abbildung 4 erlaubt es uns nicht, zu schlussfolgern, dass die niederländischen Soziologen produktiver wären als ihre belgischen Kollegen. Ich beziehe die Anzahl von Veröffentlichungen nicht auf die Anzahl von Soziologen, die in der Lage gewesen wären zu diesen Zeitschriften einen Beitrag zu leisten - denn verlässliche Listen aller Wissenschaftler die von einer belgischen oder niederländischen Organisation angestellt sind, existieren nur für die letzten Jahre. Um einen breiten Vergleich aber einigermaßen zu ermöglichen, habe ich hier alle Autoren, die in Belgien arbeiten, aufgezählt (also sowohl Autoren aus Flandern als auch aus der Wallonischen Region). 
logy, und The Netherlands' Journal of the Social Sciences. Die raison d'être dieser Zeitschrift verschwand am Ende des 20. Jahrhunderts in dem Moment, in dem mehr und mehr niederländische Autoren selbst versuchten, in internationalen Zeitschriften zu veröffentlichen.

Die >Welt $<$ der belgischen Soziologie sieht anders aus. Eine einzige internationale Zeitschrift war extrem populär bei belgischen Autoren: Social Compass. Von 1955 bis 2005 erschienen beinahe 42\% ihrer SSCI-Aufsätze in diesem Periodikum (114 der insgesamt 273). Die anderen Aufsätze waren über einen relativ weiten Bereich anderer SSCI-Zeitschriften verteilt - ohne spezifische Schwerpunkte. Social Compass ist eine bilinguale (französisch / englische) Zeitschrift im Bereich der Religionssoziologie, die den größten katholischen Universitäten in Belgien stark verbunden ist. Ihr Impact Faktor war immer niedrig; gegenwärtig steht sie auf Rang 95 von 113 soziologischen Zeitschriften. Man könnte sich daher fragen, wie global die globale Orientierung belgischer Soziologie wirklich ist oder war. Vielleicht wiederholt sich die Geschichte hier wieder: die ersten snationalen Soziologie-Zeitschriften erschienen spät in Belgien, die sglobale Orientierung ist ebenfalls etwas, das sich nur langsam in der belgischen Soziologie verankert. Dies könnte als indirekter Indikator für die Bedeutung nationaler Fachzeitschriften zur Stimulierung von intellektuellem Austausch und Kommunikation zwischen Mitgliedern wissenschaftlicher Netzwerke angesehen werden.

Die Situation der niederländischsprachigen Soziologie ist bereits mehrfach mit dem Bild eines Beobachters hinter einem Einwegspiegel beschrieben worden. Niederländische (und belgische) Soziologen nehmen wahr, was in anderen scientific communities vor sich geht, während ihre eigenen Aktivitäten von denen auf der anderen Seite des Spiegels unbe(ob)achtet bleiben. ${ }^{8}$ Gegenwärtig ist es aber schwierig zu behaupten, dass die wissenschaftliche Arbeit niederländischer Soziologen von Außenstehenden unbeobachtet bleibt. Vieles wird in vielgelesenen hochrangigen Zeitschriften veröffentlicht. Vielleicht sind die nationalen scientific communities nur ein temporäres Phänomen; nicht allein in den Natur- sondern auch in den Sozialwissenschaften. Für eine lange Zeit schien die Expansion wissenschaftlicher Netzwerke mit der Stärkung nationaler Netzwerke einherzugehen. Das nationale Setting mag eine bedeutsame Beschränkung des Kommunikationsraumes gewesen sein, vor allem für die Formierung von neuen Netzwerken wissenschaftlicher Spezialisten. Die Dynamik von Spezialisierung und disziplinärer Differenzierung scheint aber auch der Hauptgrund zu sein, warum einige nationale Settings nicht länger

8 Aber dieser Einwegspiegel war immer ein höchst selektiver. Wie aus einer Übersicht der in M\&M und SG besprochenen Bücher ersichtlich ist, erfuhr deutsche, französische und englische soziologische Literatur beachtliche Anerkennung in den Niederlanden vor dem zweiten Weltkrieg. Nach dem Krieg verschwand das niederländische Interesse an Veröffentlichungen aus der deutschen und französischen Soziologie fast vollständig. Stattdessen wurde eine - bis heute andauernde - Orientierung hin zu englischer Literatur vorherrschend (Heilbron 1982). 
ausreichende Kommunikationsmöglichkeiten für eine große Anzahl von Disziplinen und Subdisziplinen bieten. In den Niederlanden und Belgien findet die wachsende Partizipation an supranationalen Kommunikationsnetzwerken heutzutage allerdings deutlich auf Kosten nationaler Netzwerke statt.

Die nationale Struktur von wissenschaftlichen Organisationen hat die Etablierung von internationalen wissenschaftlichen Kollaborationen nicht verhindert. Deutlich ist auch, dass die Abhängigkeit der wissenschaftlichen Forschung von öffentlicher Finanzierung seit Ende des zweiten Weltkrieges nicht abgenommen hat - eher im Gegenteil. Dennoch gibt es auch beträchtliche Unterschiede zwischen den verschiedenen nationalen scientific communities. Die SoziologInnen in Belgien und den Niederlanden haben in ihren eigenen Nischen operiert; sie haben auch auf eine eigene Art und Weise auf die Globalisierung der wissenschaftlichen Kommunikation reagiert. Diese Divergenz reflektiert verschiedene wissenschaftspolitische Schwerpunkte und Akzente.

Rudolf Stichweh (2004) hat die Globalisierung und Spezialisierung der wissenschaftlichen Kommunikation stark hervorgehoben. Als Folge zunehmender Spezialisierung haben (bestimmte) nationale Netzwerke an Bedeutung eingebüßt. Solche Kommunikationsnetzwerke scheinen nicht länger eine adäquate Infrastruktur für wissenschaftliche Kommunikation zu bieten. Dafür findet man viele Beispiele. So haben sich wissenschaftliche Vereine und Fachzeitschriften mit einem rregionalen oder globalen Schwerpunkt, darunter verschiedene europäische Vereine und Zeitschriften (European Sociological Review, European Societies, European Journal of Sociology, usw.) rapide ausgedehnt. Wichtig ist auch, dass einige nationale, besonders englischsprachige Zeitschriften faktisch zu globalen Zeitschriften geworden sind (gemessen an der Zusammensetzung ihrer Herausgeber, der Herkunft ihrer Autoren und Abonnenten oder an dem impact factor), andere aber eben nicht. Wie die hier präsentierten Analysen zeigen, wirkt die Stärkung supranationaler Kommunikationsnetzwerke sich auf unterschiedliche nationale Netzwerke und ihre Zeitschriften unterschiedlich aus. Die Globalisierung der Wissenschaft erweitert nicht nur den Kommunikationsraum, sie führt auch zu neuen Strukturen und Differenzen innerhalb des Wissenschaftssystems.

\section{Schluss}

Wissenschaftliche Zeitschriften ermöglichen nicht nur die Kommunikation von Forschungsergebnissen. Sie beeinflussen gleichermaßen, in welcher Form Beiträge zu wissenschaftlicher Kommunikation geleistet werden können. Die beobachteten Möglichkeiten der Veröffentlichung beeinflussen die Auswahl von Forschungsthemen, die Spezifikation bestimmter Hypothesen, die Wahl bestimmter methodologischer Herangehensweisen, die Verbindung mit früheren Publikationen anderer Wissenschaftler (durch Zitate und 
Referenzen), usw. In einer Art Feedback-Schleife wirken Kommunikationsstrukturen und Kommunikationsmöglichkeiten auf den wissenschaftlichen Produktionsprozess selbst ein (vgl. Bazerman 1988; Stichweh 1984, $394 \mathrm{ff.;}$ Gross / Harmon / Reidy 2002). Im Anschluss an die Arbeiten von Rudolf Stichweh habe ich hier auf die Bedeutung von zwei Strukturänderungen für die Soziologie als wissenschaftliche Disziplin hingewiesen: einerseits Änderungen, die die Inklusion in Leistungsrollen (als Autor) betreffen, andererseits Änderungen, die mit der Institutionalisierung einer stärkeren globalen Orientierung einhergehen.

Die beiden Änderungen kann man in ihrem Zusammenhang betrachten. Obwohl die Nationalisierung der Wissenschaft den Kommunikationsraum der Disziplinen beschränkt, so Stichweh (2004), vergrößert sie aber gleichzeitig die Chancen der Beteiligung an wissenschaftlicher Kommunikation. Innerhalb der Grenzen nationaler scientific communities haben mehr WissenschaflerInnen und Forschungsgruppen die Möglichkeit, sich aktiv an wissenschaftlicher Kommunikation zu beteiligen. Auch der Anstieg der Beteiligung von Frauen und das Hervortreten von Formen von multiple-authorship weisen in die Richtung einer expansiven Logik. Heutzutage sind WissenschaftlerInnen in der Lage, sich als AutorInnen am wissenschaftlichen Kommunikationsprozess zu beteiligen auf Grund von erstaunlich kleinen eigenen Leistungen (in den Naturwissenschaften sind Zeitschriftaufsätze von einhundert oder mehr >Autoren mittlerweile keine Ausnahme mehr). Vor diesem Hintergrund scheint es daher kein Zufall zu sein, dass die kommunikative Relevanz der Nationalebene jetzt an Bedeutung verliert. Die Expansion institutionell anerkannter Partizipationsmöglichkeiten macht es leichter, wissenschaftliche Kommunikation mittels selektiver, globaler Kommunikationsmedien zu privilegieren.

\section{Literatur}

Bazerman, C. (1988): Shaping Written Knowledge. The Genre and Activity of the Experimental Article in Science. Madison: University of Wisconsin Press.

Clark, R. (1999): Diversity in Sociology. The American Sociologist 30, 22-41.

De Haan, J. (1997): Authorship Patterns in Dutch Sociology. Scientometrics 39, 2, 197-208.

Foucault, M. (1995): Qu'est-ce qu'un auteur? Dits et écrits I. Paris: Gallimard.

Gross, A.G. / Harmon, J. E. / Reidy, M. (2002): Communicating Science: The Scientific Article from the $17^{\text {th }}$ Century to the Present. Oxford: Oxford University Press.

Heilbron, J. (1982): Franse sociologie in Nederland: Receptiepatronen in de Nederlandse sociologie. Sociodrome 7, 4, 8-12.

Jacobs, A. / De Wit, K. (2004): Verzilvering van een collectieve inspanning: Context, ontstaan en evolutie van het Tijdschrift voor Sociologie (1980-2004). Tijdschrift voor Sociologie 25, 1, 37-71.

Keiner, E. (1999): Erziehungswissenschaft 1947-1990. Eine empirische und vergleichende Untersuchung zur kommunikativen Praxis einer Disziplin. Weinheim: Beltz.

Kuhn, Th. (1962): The structure of scientific revolution. Chicago: University of Chicago Press.

Luhmann, N. (1990): Die Wissenschaft der Gesellschaft. Frankfurt a. M.: Suhrkamp. 
Platt, J. (2007): The Women's Movement and British Journal Articles, 1950-2004. Sociology 41, 5, 961-975.

Pontille, D. (2003): Authorship Practices and Institutional Contexts in Sociology: Elements for a Comparison of the United States and France. Science, Technology, \& Human Values 28, 2, 217-243.

Stichweh, R. (1984): Zur Entstehung des modernen Systems wissenschaftlicher Disziplinen. Physik in Deutschland 1740-1890. Frankfurt a. M.: Suhrkamp.

Stichweh, R. (1987): Die Autopoiesis der Wissenschaft. S. 52-83 in: D. Baecker et al. (Hrsg.), Theorie als Passion. Frankfurt a. M.: Suhrkamp.

Stichweh, R. (1994): Wissenschaft, Universität, Professionen: Soziologische Analysen. Frankfurt a.M.: Suhrkamp.

Stichweh, R. (2003a): Genese des globalen Wissenschaftssystems. Soziale Systeme 9, 1, 13-26.

Stichweh, R. (2003b): The Multiple Publics of Science: Inclusion and Popularization. Soziale Systeme 9, 2, 210-220.

Stichweh, R. (2004): Wissensgesellschaft und Wissenschaftssystem. Schweizerische Zeitschrift für Soziologie 30, 2, 147-165.

Vanderstraeten, R. (1999): Versäulung und funktionale Differenzierung. Zur Enttraditionalisierung der katholischen Lebensformen. Soziale Welt 50, 297-314.

Wilterdink, N./van Heerikhuizen, B. (2004): Dertig jaar AST. Amsterdams Sociologisch Tijdschrift 31, 4, 423-430.

Prof. Dr. Raf Vanderstraeten

Center for Social Theory, Department of Sociology

Ghent University

Korte Meer 3-5, BE-9000 Gent, Belgien,

Raf.Vanderstraeten@UGent.be 